\title{
Open Heavy Flavor Production at Forward Angles in PHENIX
}

\author{
K.F. Read for the PHENIX Collaboration ${ }^{1}$ \\ Oak Ridge National Laboratory, Oak Ridge, TN 37831, USA
}

\begin{abstract}
The measurement of the nuclear modification factor $\left(R_{\mathrm{AA}}\right)$ for heavy-flavor production in heavy-ion collisions tests predictions for cold- and hot-nuclear-matter effects. Heavy-flavor production in $p+p$ collisions tests $\mathrm{PQCD}$ calculations and serves as a reference for understanding heavy-flavor production in heavy-ion collisions. Using the PHENIX muon-arm spectrometers, the transverse momentum spectra of inclusive muon candidates are measured for $p+p$ and $\mathrm{Cu}+\mathrm{Cu}$ collisions at $\sqrt{s_{\mathrm{NN}}}=200 \mathrm{GeV}$. After subtracting backgrounds, we obtain the measured invariant yields of negative muons from the decay of heavy flavor mesons. For $p+p$ collisions, we measure the charm-production cross section integrated over $p_{T}$ and in the rapidity range $1.4<y<1.9$ to be $d \sigma_{c \bar{c}} / d y=0.139 \pm 0.029$ (stat) ${ }_{-0.058}^{+0.051}$ (syst) mb. This result is compared to a recent FONLL calculation and to a PHENIX measurement at mid-rapidity. For $\mathrm{Cu}+\mathrm{Cu}$ collisions, we measure the $R_{\mathrm{AA}}$ for heavy-flavor muons in three centrality bins for $1<p_{T}<4 \mathrm{GeV} / c$, with suppression observed for central collisions. We compare our measurement for central collisions to a recent theoretical prediction.
\end{abstract}

Keywords: PHENIX, heavy ions, heavy flavor

\section{Introduction}

Heavy quarks are produced in the early stages of heavy-ion collisions and, therefore, are a probe of the hot dense partonic matter produced. Accurate measurements serve as a critical test and constraint for predictions concerning the energy loss mechanism of partons moving through such novel nuclear matter. This remains a priority for the field of heavy-ion collision physics today. Measurement of heavy quark production in $p+p$ collisions tests perturbative Quantum Chromodynamics (pQCD) calculations, in addition to serving as a reference for understanding production in heavy ion collisions.

The nuclear modification factor $R_{\mathrm{AA}}$ for $\mathrm{Cu}+\mathrm{Cu}$ collisions using a $p+p$ reference is defined to be

$$
R_{\mathrm{AA}}=\frac{1}{\left\langle N_{\mathrm{coll}}\right\rangle} \frac{d^{2} N_{\mathrm{Cu}+\mathrm{Cu}} / d p_{T} d \eta}{d^{2} N_{p p} / d p_{T} d \eta},
$$

where $\left\langle N_{\text {coll }}\right\rangle$ is the average number of nucleon-nucleon collisions in the $\mathrm{Cu}+\mathrm{Cu}$ collision based on a simple description of the nucleus [1], averaged over a given centrality bin. Collisions are classified experimentally according to centrality [1], the percentage of the total nuclear inelastic cross section, with $0 \%$ representing the most head-on collisions.

Email address: readkf@ornl .gov (K.F. Read for the PHENIX Collaboration)

${ }^{1}$ Research sponsored by the Office of Nuclear Physics, U.S. Department of Energy 

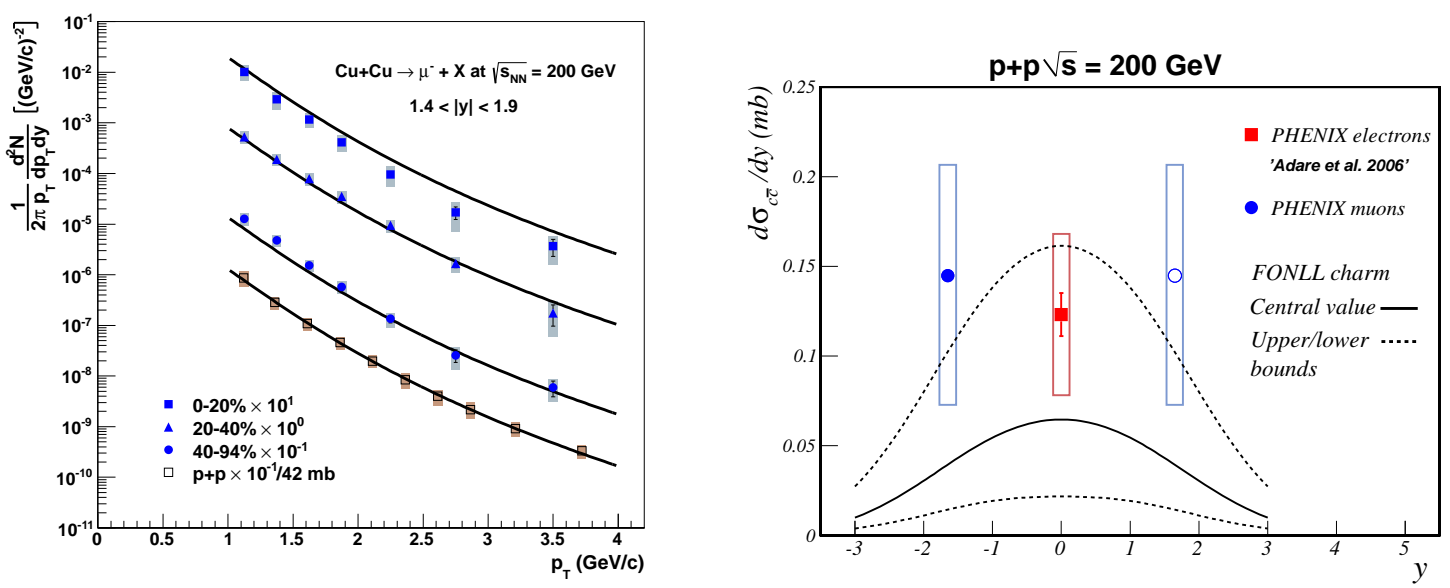

Figure 1: Left: Invariant yields of negative muons produced from heavy-flavor meson decay as a function of $p_{T}$ in $p+p$ collisions at $\sqrt{s}=200$ $\mathrm{GeV}$ (open squares) and in $\mathrm{Cu}+\mathrm{Cu}$ collisions at $\sqrt{s_{\mathrm{NN}}}=200 \mathrm{GeV}$ for three different centrality bins (40-94\%, 20-40\% and 0-20\%), scaled by powers of ten for clarity (filled symbols) [2]. Solid lines are a fit to the $p+p$ yield described in the text [3], scaled by the corresponding average number of binary collisions $\left\langle N_{\text {coll }}\right\rangle$. Right: $c \bar{c}$ production cross section as a function of rapidity in $p+p$ collisions at $\sqrt{s}=200 \mathrm{GeV}$, measured using semileptonic decay muons at forward rapidity (circles) [2] and electrons at mid-rapidity (square) [4]. Curves represent a theoretical prediction [5] 6].

In the absence of nuclear effects, $R_{\mathrm{AA}}$ is expected to be equal to unity. Values less than unity are referred to as "suppression." Heavy quark production is expected to be modified by cold-nuclear matter effects, such as shadowing and initial-state energy loss, which can effect $R_{\mathrm{AA}}$. It is important to complement existing measurements for open heavy flavor production at mid-rapidity with measurements at forward rapidities to fully test theoretical models.

\section{Methodology}

This analysis is based on measurements by the PHENIX Experiment of $p+p$ and $\mathrm{Cu}+\mathrm{Cu}$ collisions at a center-ofmass energy per nucleon-nucleon collision of $\sqrt{s_{\mathrm{NN}}}=200 \mathrm{GeV}$. The south and north PHENIX muon arm spectrometers cover the pseudorapidity range $-2.2<\eta<-1.2$ and $1.2<\eta<2.4$, respectively, over the full azimuth. Each muon arm contains a Muon Identifier (MuID) consisting of interleaved planes of steel absorber and Iarocci tubes, with the five instrumented "gaps" numbered from 0 to 4 . Beam-Beam Counters (BBC), composed of arrays of quartz Čerenkov detectors on either side of the interaction point, provide a minimum bias trigger.

The double differential invariant yield for muons from the semileptonic decay of heavy flavor mesons is:

$$
\frac{d^{2} N^{\mu}}{2 \pi p_{T} d p_{T} d \eta}=\frac{1}{2 \pi p_{T} \Delta p_{T} \Delta \eta} \frac{N_{I}-N_{C}-N_{F}}{N_{\mathrm{evt}} \epsilon_{\mathrm{BBC}}^{c \bar{c} \rightarrow \mu} A \epsilon}
$$

where $N_{I}$ is the total number of muon candidates in the bin that reach the last gap of the MuID (Gap 4) and pass all track selection criteria; $N_{C}$ is the number of tracks corresponding to the irreducible hadronic background estimated using a hadron cocktail; $N_{F}$ is the estimated number of surviving misreconstructed tracks; $N_{\text {evt }}$ is the number of events; $A \epsilon$ is the detector acceptance and efficiency correction; and $\epsilon_{\mathrm{BBC}}^{c \bar{c} \rightarrow \mu}$ is the BBC trigger efficiency for events with a heavy-flavor muon at forward rapidity. The signal-to-background ratio is better for negative muons than positive muons because antiprotons and negative kaons are more suppressed than their positive counterparts by the Muon Tracker front absorbers. Therefore, only negative muon candidates (and background estimates) are considered in this analysis [2].

A data-driven Monte Carlo "cocktail" is used to predict/simulate the irreducible hadronic background $N_{C}$. The cocktail consists of a collection of individual simulated primary hadrons which are weighted to match RHIC data. Specifically, the transverse momentum spectrum for neutral pions is adjusted to agree with PHENIX central arm measurements extrapolated to our rapidity region. Other hadrons are thrown so that they conform to hadron-to-pion 

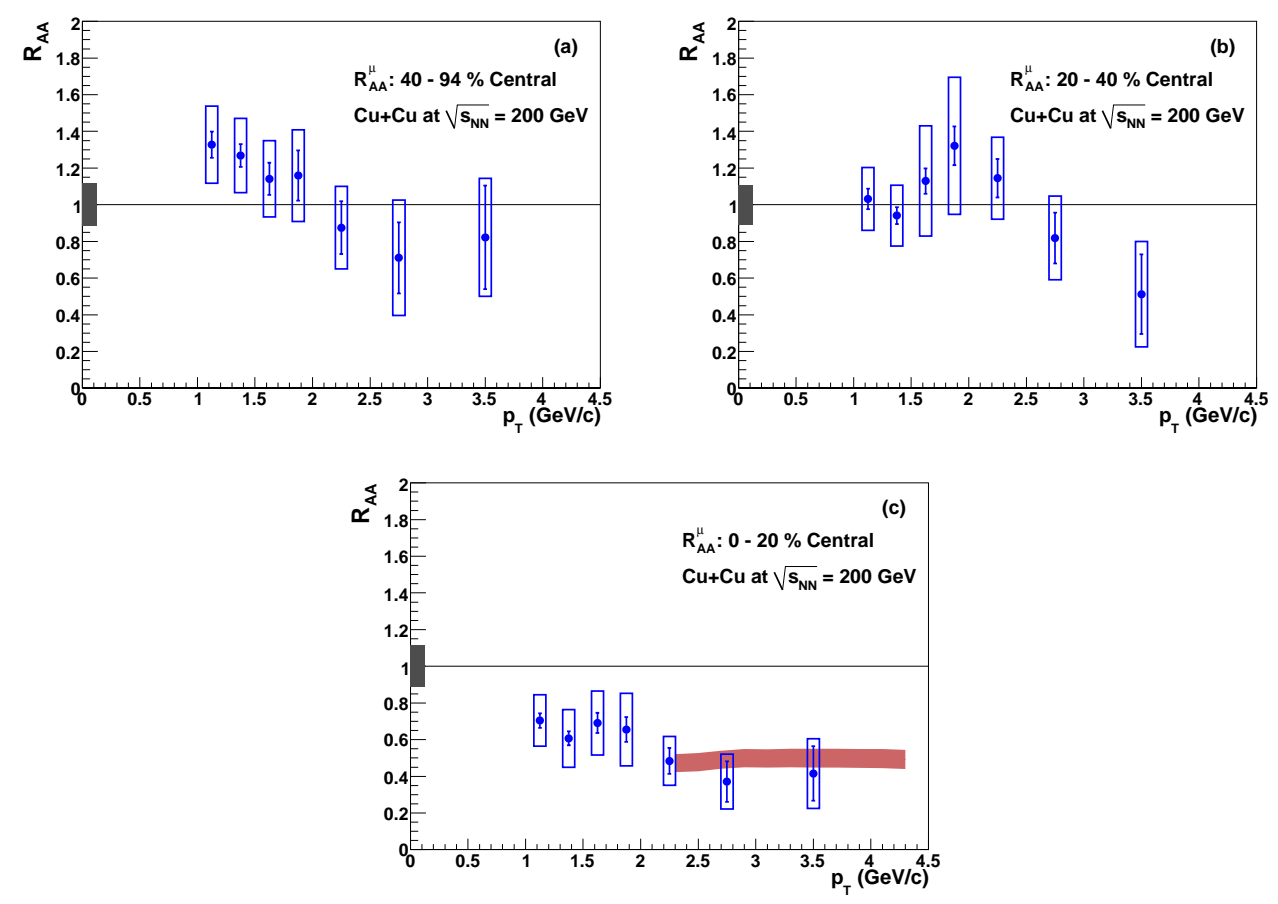

Figure 2: (Color online) Transverse momentum distribution of $R_{\mathrm{AA}}$ for negative muons from heavy-flavor meson decay in $\mathrm{Cu}+\mathrm{Cu}$ collisions at $\sqrt{s_{\mathrm{NN}}}=200 \mathrm{GeV}$ for the centrality bins: $40-94 \%$ (a), 20-40\% (b), and 0-20\% (c) [2]. The band in (c) is a theoretical prediction from [7] 8].

ratios measured by experiments at RHIC. In order to account for any degradation of reconstructed track quality in the $\mathrm{Cu}+\mathrm{Cu}$ analysis, we embed the hadron cocktail tracks in real $\mathrm{Cu}+\mathrm{Cu}$ events. The "cocktail" is propagated via GEANT through a complete simulation of the PHENIX muon arms. In order to explore sensitivity to the simulated propagation through the thick material of the MuID, we produced background estimates using both the FLUKA and GHEISHA hadron shower codes, with a range of modified hadron-iron interaction cross sections [2]. Tracks which stop at MuID Gap 2 or 3 with no hit further downstream (Gap 3 or 4, respectively) have a characteristic distribution of longitudinal momentum. Decay muons exhibit a sharp peak due to electromagnetic energy loss, but hadrons exhibit a broad shoulder. A highly purified sample of hadrons can be obtained from such tracks by requiring $p_{z}>3 \mathrm{GeV} / \mathrm{c}$. The reliance on initial distributions for the hadron cocktail is significantly minimized by subsequent "tuning" which further adjusts the hadron weights to match the measurements for hadrons from MuID Gap 2 and Gap 3.

Simulation studies show that the number of decay muons that reach MuID Gap 4 linearly increases with the longitudinal distance of the vertex from the respective muon arm. Hadrons do not exhibit this dependence. The inclusive sample of tracks reaching MuID Gap 4 will exhibit a linear dependence of $d N_{\mu} / d z_{\mathrm{BBC}}$ on $z_{\mathrm{BBC}}$, the event vertex longitudinal position measured by the BBC. The weights of the hadron cocktail can be adjusted to match this slope for each arm for different transverse momentum bins.

The number of tracks in the hadron cocktail that satisfy all selection criteria is $N_{C}$, which we subtract from the inclusive yield $N_{I}$ as indicated in Equation 2. After correcting for acceptance and efficiencies, we obtain the measured invariant yield of negatively charged muons from the decay of heavy-flavor mesons in $p+p$ collisions at $\sqrt{s}=200 \mathrm{GeV}$ and for three centrality bins of $\mathrm{Cu}+\mathrm{Cu}$ collisions at $\sqrt{s_{\mathrm{NN}}}=200 \mathrm{GeV}$. In order to obtain the nuclear modification factor and fully exploit the cancellation of common systematic errors, we use Equation 1 for each bin of transverse momentum and for a common hadron cocktail optimization. This is possible in an experiment which measures its own reference data for computation of a nuclear modification factor. 


\section{Results}

The measured invariant yields of negatively charged muons from the decay of heavy-flavor mesons in $p+p$ collisions at $\sqrt{s}=200 \mathrm{GeV}$ and for three centrality bins of $\mathrm{Cu}+\mathrm{Cu}$ collisions at $\sqrt{s_{\mathrm{NN}}}=200 \mathrm{GeV}$ are shown in the left panel of Figure 1 scaled by powers of 10 for clarity. The solid lines are a fit to the $p+p$ yield using the function $A\left[1+\left(p_{T} / B\right)^{2}\right]^{-4.2}$ based on [3], scaled by the corresponding average number of binary collisions $\left\langle N_{\text {coll }}\right\rangle$ for the $\mathrm{Cu}+\mathrm{Cu}$ measurements. The contribution from bottom meson decay is estimated to be negligible for this transverse momentum region. In order to estimate the full charm cross section for $p+p$ collisions, we express the $p+p$ invariant yield as a differential cross section $d \sigma_{\mu^{-}} / d y$ after extrapolating the measurement to $p_{\mathrm{T}}=0 \mathrm{GeV} / \mathrm{c}$ using fixed-orderplus-next-to-leading-log (FONLL) [5, 6] calculations. The charm-production cross section $d \sigma_{c \bar{c}} / d y$ is then computed using:

$$
d \sigma_{c \bar{c}} / d y=\frac{1}{B R(c \rightarrow \mu)} \cdot \frac{1}{C_{l / D}} \cdot \frac{d \sigma_{\mu^{-}}}{d y}
$$

where $B R(c \rightarrow \mu)$ is the total charm to muon branching ratio and $C_{l / D}$ is a kinematic correction factor based on the FONLL calculation. We obtain a charm-production cross section integrated over $p_{T}$ and in the rapidity range $1.4<y<1.9$ of $d \sigma_{c \bar{c}} / d y=0.139 \pm 0.029$ (stat) ${ }_{-0.058}^{+0.051}$ (syst) mb. The right panel of Figure 1 shows the $c \bar{c}$ production cross section as a function of rapidity using this measurement of muons at forward rapidity [2] compared to a PHENIX measurement using electrons at mid-rapidity [4].

Figure 2 shows the transverse momentum distribution of $R_{\mathrm{AA}}$ for negative muons from heavy-flavor meson decay in $\mathrm{Cu}+\mathrm{Cu}$ collisions for three centrality bins [2]. The (red) band in Figure 2] shows a recent theoretical prediction [7, 8] for $y=1.65$ and $p_{T}>2.5 \mathrm{GeV} / \mathrm{c}$ which includes the effects of heavy quark energy loss, in-medium heavy meson dissociation, and cold nuclear matter effects such as shadowing and initial state energy loss of the incoming partons.

\section{Discussion and Conclusions}

Negative muons from the decay of heavy flavor mesons in $p+p$ collisions at $\sqrt{s}=200 \mathrm{GeV}$ are used to measure the charm-production cross section integrated over $p_{T}$ and in the rapidity range $1.4<y<1.9$. The result is $d \sigma_{c \bar{c}} / d y=$ $0.139 \pm 0.029$ (stat) ${ }_{-0.058}^{+0.051}$ (syst) mb. This result is compatible with a FONLL calculation within experimental and theoretical scale uncertainties. Negative muons from heavy-flavor meson decay have also been measured in $\mathrm{Cu}+\mathrm{Cu}$ collisons at $\sqrt{s_{\mathrm{NN}}}=200 \mathrm{GeV}$ for the same rapidity and momentum range, and for 3 bins of centrality. Reasonable agreement is observed between the invariant yields for the peripheral (40-90\%) and mid-central (20-40\%) collisions and scaled fits to the invariant yield for $p+p$ collisions; however, a suppression is observed at higher $p_{T}$ for the central $(0-20 \%) \mathrm{Cu}+\mathrm{Cu}$ collisions. This effect is quantified by the reported nuclear modification factors $R_{\mathrm{AA}}$ measured in three centrality bins. We observe significant suppression of heavy-flavor muon production in $\mathrm{central} \mathrm{Cu}+\mathrm{Cu}$ collisions $(0-20 \%)$, with the largest effect for $p_{T}>2 \mathrm{GeV} / \mathrm{c}$. The measurement for central collisions is consistent with a recent theoretical prediction for this rapidity and transverse momentum range [7, 8].

The PHENIX Experiment has installed new inner silicon vertex detectors which will allow separation of charm and bottom contributions in future measurements. Such measurements will constrain predictions and help guide theoretical understanding as part of an improving understanding of QCD matter at extreme energy densities.

\section{References}

[1] M. L. Miller, K. Reygers, S. J. Sanders, P. Steinberg, Ann. Rev. Nucl. Part. Sci. 57 (2007) 205-243. doi:10.1146/annurev.nucl.57. 090506.123020

[2] A. Adare, et al., Phys. Rev. C 86 (2012) 024909.

[3] J. Yoh, et al., Phys. Rev. Lett. 41 (1978) 684.

[4] A. Adare, et al., Phys. Rev. Lett. 97 (2006) 252002. doi:10.1103/PhysRevLett.97.252002

[5] M. Cacciari, M. Greco, P. Nason, JHEP 9805 (1998) 007.

[6] M. Cacciari, S. Frixione, P. Nason, JHEP 0103 (2001) 006.

[7] I. Vitev, private communication (2011).

[8] R. Sharma, I. Vitev, B.-W. Zhang, Phys. Rev. C 80 (2009) 054902. doi : 10.1103/PhysRevC.80.054902 